A N N A L E S Annales de Bretagne et des Pays de l'Ouest

\title{
Claude Gauvard, Violence et ordre public au Moyen Âge
}

\section{Franck Mercier}

\section{(2) OpenEdition}

\section{Journals}

Édition électronique

URL : http://journals.openedition.org/abpo/950

DOI : 10.4000/abpo.950

ISBN : 978-2-7535-1501-7

ISSN : 2108-6443

\section{Éditeur}

Presses universitaires de Rennes

Édition imprimée

Date de publication : 30 mars 2006

Pagination : 167-169

ISBN : 978-2-7535-0273-4

ISSN : 0399-0826

\section{Référence électronique}

Franck Mercier, "Claude Gauvard, Violence et ordre public au Moyen Âge », Annales de Bretagne et des Pays de l'Ouest [En ligne], 113-1 | 2006, mis en ligne le 30 mars 2008, consulté le 23 septembre 2020. URL : http://journals.openedition.org/abpo/950 ; DOI : https://doi.org/10.4000/abpo.950 
Nominoé en Poher plutôt qu'en Vannetais, en localisant son aula de "Botnumel " à Botmel [en Callac]. Il ajoute toutefois que le chef breton est donné comme comte de Vannes dès avant 830, en s'appuyant sur les chartes 252 (6 juin 827?) et 250 (820-830?) de Redon. Mais, d'une part, Bernard Tanguy identifie " Botnumel " avec Bonnevel en Priziac, sur le haut Ellé, là même où une quinzaine d'années plus tôt Louis le Pieux a vaincu Morvan (ce qui renforce paradoxalement l'hypothèse d'Hubert Guillotel). D'autre part, dans l'édition récente en fac-simile du Cartulaire de Redon ${ }^{1}$, H. Guillotel avait lui-même revu les datations jusqu'alors proposées pour les deux chartes en question. Il plaçait l'une en 832 et l'autre en 834, ce qui invalide l'objection soulevée par H. Pettiau. Notons aussi au passage que Judicael " rex britonnum " est contemporain de Dagobert I ${ }^{\text {er }}$ (629-638) et non de Dagobert II (676-679), comme une coquille pourrait ici le laisser à penser.

Ces réserves mineures n'empêcheront pas ce travail de rendre d'incontestables services aux chercheurs qui trouveront commodément rassemblées et critiquées une masse d'informations jusqu'alors fort dispersées. Il est à la fois significatif et réconfortant de voir la Bretagne prendre ainsi la place qui lui revient dans une revue internationale destinée à embrasser l'ensemble des pays celtiques.

Bernard MERDRIGNAC

GaUvard, Claude, Violence et ordre public au Moyen Âge, Paris, Picard, 2005.

L'œuvre de Claude Gauvard est marquée par la passion de comprendre le rôle de la criminalité et de la justice dans la construction de l'État à la fin du Moyen Âge. En hommage à celle-ci, les éditions Picard viennent de publier, dans la collection des 7Médiévistes français, un important recueil de ses articles parus dans diverses revues spécialisées entre 1989 et 2000. L'ouvrage fait ainsi le bilan de dix ans de recherches consacrées à la violence et à la justice dans la France des XIV et $\mathrm{XV}^{\mathrm{e}}$ siècles. Si la place éminente de Claude Gauvard dans le champ de l'historiographie médiévale nous dispense de la présenter, la réunion en un seul ouvrage d'une quinzaine de ses articles récents fait mieux ressortir la cohérence et l'originalité de son approche de l'ordre et du désordre à la fin du Moyen Âge. C'est bien du reste le but de cette collection que de faire connaître les travaux (parfois difficiles d'accès) des grands noms de la médiévistique française. Divisé en quatorze chapitres (correspondant à autant d'articles indépendants), l'ouvrage s'organise autour de trois sections générales ("crimes et modes de résolution ", " juges et jugements ", "violences et contrôle social "). Un habile montage emboîte ainsi des articles relativement pointus portant sur des thèmes ou des affaires précises (comme par exemple l'assassinat de Guillaume de Flavy ou le procès de Guillaume de Tignonville) dans un discours plus synthétique sur la violence (le volume s'ouvre par l'article "violence " du Dictionnaire raisonné de l'Occident médiéval).

D'un chapitre à l'autre, se découvrent ainsi les lignes maîtresse d'une ample réflexion sur la violence et la justice qui entend bien se tenir à distance de deux positions historiographiques également contestables et contestées : la première

1. Il convient de signaler la parution d'un DVD reprenant la teneur des 2 vol. édités par l'AHID en 1998 et 2004. 
consiste à stigmatiser la violence spontanée inhérente à "l'homme médiéval ", la seconde à projeter inconsidérément sur les deux derniers siècles du Moyen Âge le schéma moderne de l'éclat des supplices. C'est contre cette double vision ensauvagée et terrorisante du Moyen Âge, que Claude Gauvard développe ses analyses autour de la place du crime dans la société médiévale.

Il s'agit d'abord de battre en brèche les préjugés qui tendent à associer le Moyen Âge à la violence débridée d'avant le polissage de la " civilisation des moeurs " (préjugés qui continuent parfois à faire des ravages dans une historiographie imbue des théories de Norbert Elias). Si la violence demeure bien " l'un des ressorts essentiels de la société médiévale " (p. 16), elle n'est cependant pas spontanée ni sauvage. Aujourd'hui comme hier, il n'existe pas de violence gratuite, mais seulement des formes de violence que l'on ne comprend pas ou mal. Reconnaissant dans la société médiévale une société à honneur, telle que la définissent les anthropologues, l'auteur s'emploie à comprendre le surgissement perturbateur de la violence comme une forme de défense de l'honneur. La société médiévale manifeste en effet une assez grande tolérance à l'égard de l'homicide dès-lors que celui-ci trouve son origine dans l'honneur blessé, d'où la difficulté apparente à inscrire l'homicide dans le champ pénal à la fin du Moyen Âge. L'emprise sociale de ce code de l'honneur (qui n'est pas propre à la noblesse mais transcende tous les clivages sociaux) serait si forte qu'elle contaminerait l'appareil judiciaire de l'État naissant. Le roi, en effet, " partage avec l'opinion ces valeurs qui placent encore l'honneur au-dessus du respect de la personne humaine " (p. 83). On pourra certes reprocher à Claude Gauvard de voir l'honneur partout défendu, chez les nobles, comme chez le roi puisque la promotion de l'honneur s'effectue jusque dans l'enceinte du Parlement de Paris où la justice royale déléguée peine à imposer sa propre conception de l'ordre public face à une noblesse prompte à se venger. Cette approche globale de la criminalité, nourrie des apports de l'anthropologie, a cependant le mérite de ramener le regard de l'historien vers le centre de la société, là où se nouent et se dénouent véritablement les conflits personnels et collectifs. Pour Claude Gauvard, la violence usuelle ne se trouve pas là où on l'attend le plus, soit aux marges du tissu social, mais s'inscrit bien plutôt au cœur des " relations conflictuelles que des hommes ordinaires peuvent avoir entre eux " (p. 16). À travers le rôle de la justice royale à la fin du Moyen Âge, ce recueil nous plonge aussi d'emblée au cœur d'un ambitieux projet historiographique qui vise à " comprendre le lien entre le développement de la justice et la naissance de l'État " (p. 80).

C'est ici que Claude Gauvard s'inscrit en faux contre la vision terrifiante, purement coercitive, de la justice médiévale encore trop souvent véhiculée par l'imaginaire contemporain. Tout en admettant l'action punitive exemplaire de la justice, l'auteur préfère en effet insister sur la dimension miséricordieuse du pouvoir royal qui sait tout autant, sinon mieux, pardonner que punir. "Les deux derniers siècles du Moyen Âge restent bien le temps vivant de la grâce royale " (p. 82) à laquelle aucun crime, même ceux théoriquement définis comme irrémissibles, ne résiste. Le " pouvoir justicier qui se développe alors est loin de se muer en un pouvoir coercitif absolu et son arme semble moins la quête rigoureuse d'un aveu conduisant au gibet que l'octroi de la grâce sous la forme de la lettre de rémission " (p. 79). Dans cette perspective, la tendance commune du peuple et du roi à refouler la criminalité ressentie (à distinguer de la criminalité réelle) aux marges de la société sur la tête des professionnels du crime, à commencer par les hommes d'armes (p. 281) ménage la possibilité d'une rencontre (voire d'une entente) entre les deux acteurs au carrefour, précisément, de la justice. Il reste que dans ce triomphe de la grâce royale subsistent des zones d'ombres que l'au- 
teur est amenée aussi à repérer, sinon vraiment à explorer : force, par exemple, est bien de constater que le temps vivant de la grâce (plus particulièrement identifié au règne de Charles VI) cö̈ncide avec un durcissement ponctuel de la justice princière sous le gouvernement des Marmousets ou celui de Louis d'Orléans. De fait, s'il s'agit bien aussi de saisir " la véritable nature du pouvoir justicier en France à la fin du Moyen Âge " (p. 81), il ne faudrait peut-être pas non plus radicalement opposer la politique de la grâce à celle de la peine de mort mais les envisager de façon complémentaire, comme les deux faces d'un même pouvoir qui, de façon souveraine, peut décider de la vie ou de la mort. Le constat dressé ici (par exemple dans la conclusion, p. 273) de l'extrême rareté de l'application de la peine de mort à l'échelle du royaume n'empêche pas de relever ailleurs (chapitre 6) que Paris à la fin du XIV e siècle " devient un centre des exécutions capitales ". Cette exception parisienne suggère bien l'existence, à proximité immédiate du pouvoir (c'est-à-dire à Paris, dans l'entourage du roi ou dans les hautes sphères de la pensée politique) d'une réflexion doublée parfois d'une application rigoureuse de la justice : que l'on pense aux prévôts à poigne du tournant du siècle, Hugues Aubriot ou Guillaume de Tignonville auxquels sont d'ailleurs consacrés des pages fort intéressantes! Du reste, Claude Gauvard admet aussi que ce recentrage du regard historien sur la violence ordinaire ne doit pas non plus faire oublier le rôle important des marges dans la définition des normes et le renforcement du pouvoir. La criminalité des marges joue un rôle important à la fin du Moyen Âge, moins en raison de son importance réelle qu'en ce qu'elle soutient et justifie un discours d'exclusion de la part de ceux-là même (les " hommes ordinaires ") qui n'hésitent pas à vider leur querelle dans le sang (p. 102-109). Il reste que la vision privilégiée de la justice royale (celle du pardon miséricordieux), pour être plus proche de la réalité statistique, ne l'est pas forcément de la réalité politique. Certes, la peine de mort est loin d'être systématiquement prononcée et appliquée par les tribunaux royaux. Et ce rappel peut contribuer à relativiser l'image construite (par une certaine historiographie) d'une justice implacable, adepte de l'éclat des supplices. L'arsenal des peines dont use ou que réclame le Parlement de Paris ne correspond pas à ce musée des supplices trop souvent imputé, à tort, à la justice médiévale (chapitre 9). De là à considérer le Parlement comme " une sorte de forum où s'échangent et se classent les valeurs [...] sur lesquels se fonde le corps socio-politique " (p. 174), il y a un pas que tous les historiens ne seront peut-être pas disposés à franchir. À la grande différence du Parlement anglais, la cour d'appel royale reste cantonnée dans une activité purement judiciaire et la "négociation " des valeurs dont elle serait à la fois le lieu et l'instrument semble pour le moins biaisé. Quelle est vraiment la nature de ce " dialogue entre le roi et le peuple " dont la justice royale serait le lieu par excellence? La question mériterait sans doute d'être approfondie au plan politique. Il reste en effet que l'affirmation de la grandeur souveraine du Prince passe également, en cette fin du Moyen Âge, par la répression des crimes qui la mettent en cause. De ce point de vue, il conviendrait peutêtre aussi de s'interroger sur les différentes formes de violence d'État qui, pour être moins ordinaires, n'en participent pas moins (mais par le moyen de l'exception justement) à la construction de l'État moderne, un État fondé sur un lien politique sans aucun doute plus proche de la sujétion que de la réciprocité de l'échange entre le peuple et le roi. 